\title{
Cost-Effective Resource Allocation of Overlay Routing Relay Nodes - A Survey
}

\author{
Anuradha B. Jadhav ${ }^{1}$, Nandkishor G. Dharashive ${ }^{2}$ \\ ${ }^{1}$ SRTM University, Department of CSE, M. S. Bidve Engineering College, Latur, Maharashtra, India \\ ${ }^{2}$ Assistant Professor, Department of CSE, M. S. Bidve Engineering College, Latur, Maharashtra, India,
}

\begin{abstract}
Today Internet is a very popular and vastly growing application. It added support for various new services as compared to past 3 decades Internet. The services like mobility, real time support and much more requires continuous evolution in Internet techniques and infrastructure. As number of users increasing day by day, Internet suffers from various problems such as bottleneck, congestion, path and server failure etc. To overcome or minimize these problems Internet wants new schemes with little or no change in present routing infrastructure. Overlays are the best solution; which provides new functionalities and improves previous properties without making any change in routing standards and network infrastructure. Overlay is an additional virtual layer providing new functionalities to present routing infrastructure. Overlay network constructed on top of surviving network. Some present overlay networks are oTCP, RON, Detour, G-ISP designed to provide some functionalities. oTCP is an Overlay network, it increase TCP performance of connection by converting a TCP connection into multiple pipelined sub-connection. Each sub-connection follows end to end nature of TCP. RON is designed to provide fast fault recovery. RON is an application layer overlay network so it route packet according to application specific routing metrics. Instead of dedicated links Detour takes alternate paths to increase performance and availability. Detour routers form a virtual layer of functionality by tunneling packets over standard Internet. The inter-domain routing functionalities such as end to end inter-AS QoS, multicasting are offered by G-ISP to its customers, which today's Internet doesn't provide.
\end{abstract}

Keywords: Overlay, Overlay network

\section{Introduction}

Internet is most useful example of internetworking. Its popularity rises gradually. Daily thousands of new users join Internet. The Internet's scale, heterogeneity and dynamic nature make it useful all over the world. At the same time these features make it difficult to find exact cause of performance problems [5]. Failures like link failure and router failure are often in Internet. To overcome these failures, routing protocol takes seconds to several minutes time. This convergence time is work as an outage for certain end to end connection. This degrades the Internet performance. Without modifying the infrastructure of Internet, Overlay routing provides an effective way to improve reliability and efficiency of Internet, which is emerged in recent few years.

In Overlay network end host may locate at different ASs can perform as Overlay nodes. Thus an Overlay network is a virtual network of nodes and logical links built on top of existing network with the purpose to implement a new service and enhance performance which is not available in existing network. Underlying network uses various services provided by Overlay for effective resource utilization. Overlay routing is an application level routing mechanism that provides application level routes for network application traffic and improves user perceived network performance, such as end to end latency and available bandwidth, without modifying the current IP network [4].

One Overlay link contains one or more physical links. When a default path is affected by the failure (such as link failure or performance degradation), the source can reroute the traffic via a strategically placed relay node to words the destination [6]. Overlay choose route according to metrics applied for the path, such as end to end latency, packet loss ratio, available bandwidth, and TCP throughput. Path outage can overcome by Overlay nodes present in the network. In short duration they also bypass congestion. In Overlay routing, an end host has the capability to route the traffic to its destination through one or more Overlay relay nodes [4].

To form the Overlay network we have to select some nodes from underlying network which are called as Relay nodes or Overlay nodes. There is a huge impact of Overlay node deployment on the network performance. If we consider every node of underlying network as Overlay node, it complicates the network performance. So to optimize the number of Overlay nodes, we must strategically choose the nodes from underlying network, to act as relay nodes. To avoid the inefficiencies and limitations in network level routing, intelligent deployment of Overlay nodes is necessary. Extending the capacity of existing Overlays requires re-wiring of the network to fulfill the changing conditions, and which is not cost effective [2]. Depending on service provided various examples of overlays areoTCP, RON, Detour, G-ISP are presented.

\section{Related Systems}

\section{1 оTCP}

oTCP (Overlay TCP) [3] is a general purpose application level protocol and act as extension to TCP. A TCP connection with large RTT is splits into sub connections with small RTTs by oTCP to increase TCP throughput using overlay routing. A characteristic that increases the performance of TCP is that, the average RTT is inversely proportional to the window size and window size is directly proportional to the performance. That means if we reduce 


\section{International Journal of Science and Research (IJSR) ISSN (Online): 2319-7064 \\ Index Copernicus Value (2013): 6.14 | Impact Factor (2014): 5.611}

the RTT between connection endpoints it increases throughput by increasing window size.

An effective way to reduce the RTT of a direct connection between two hosts is to break the direct connection into multiple sub connections, in which each sub connection have the RTT smaller than RTT of direct connection. The peer nodes work as the intermediaries to split the TCP connection to increase the end to end performance. It increases throughput of direct connection as well as it detect failures and discover better alternate paths. That means it enhances reliability and quality of path between end points.

oTCP doesn't change the behavior of TCP as it built up on top of unchanged TCP protocol. Detour and RON are the schemes which are based on end to end working overlays. But oTCP is different from these as it breaks a connection in to transport layer not in network layer to reduce RTT. The sum of RTTs of sub connection is greater than RTT of direct connection then also the TCP throughput is improved. oTCP doesn't follow end to end nature of TCP connection. As ACK signal is now reception at intermediary and not at final destination. As each sub connection is a TCP connection, status of one end is replicated at other end and resources are shared fairly so that applications using oTCP are unaffected.

\subsection{RON}

The nodes present in various routing domains comes together to form a RON (Resilient Overlay Network) [4]. Whether communicating RON node fall down or underlying network facing path redundancy problem in both cases RON able to communicate by means of using other RON nodes or by finding other communicating paths. In RON aggressive probing and passive monitoring of paths is used to detect problems between its connected nodes. Always best path is searched for data sending whether it is a direct path or a virtual link goes through RON nodes. Routing protocol exchange path quality information between RON nodes, this information helps to build forwarding tables. These forwarding tables are based on various path metrics information such as latency, packet loss rate and available throughput. In RON the distributed applications can able to select path and route the packets. RON provides some facilities to classify packets in to categories to decide whether the packet is accepted or rejected. RON separate out scalability and fault detection and recovery operation from each other. The scalability operation is handled by underlying network and fault detection and recovery is performed by higher layer overlay so that can give fast reply. RON overcomes path outage and performance failure in few seconds by using path redundancy of underlying network. Each RON is closely tied to the application using it so it is called as an application controlled routing overlay. RON use application specific path metrics and policies for selecting paths.

The information exchanged by routing protocol between RON nodes is used to discover the topology of overlay network. RON client is a program running on each node communicating with RON software. Conduit is an API used for communicating between RON and RON client. The node where packet enters in RON is called as entry node and last delivery node is called as exit node. A RON header with flow identifier is tagged by entry node to the packet to identify the flow of packet. Each RON node keeps information about three specific metrics such as latency, packet loss rate and throughput for each virtual link. RON nodes are connected in complete graph as in N-node RON each node has N-1 virtual links. And each RON node routes periodically requests for different performance metrics. When a RON node has insufficient information about other node that means the other RON node is unavailable completely. RON user or administrator can define the type of traffic passes through a particular link in network.

\subsection{Detour}

Detour [5] is a virtual Internet on top of actual physical network substrate. In Detour instead of using dedicated Internet links packets are ,tunnel ${ }^{\text {ee }}$ by routers over the commodity Internet. This design is used to overcome the performance and availability problems of Internet. Detour enhances the end to end application performance by forwarding packets through alternate path instead of default paths. This will also minimize the bottleneck problem or congestion problem occurs on default link. Routers spread load unequally while using some links totally and leaving either links unused. In that case Detour tries to take alternate paths to control load balancing. Detour router exchange connectivity as well as performance information such as latency, packet drop rate. Detour is a new virtual network present on top of the existing Internet. The routing system of Detour takes real Internet links and traffic as input to explore alternate paths for hosts and network solutions.

In Detour geographically distributed routers are grouped as a set and interconnected using tunnels. Tunnel is a virtual link connecting two points. A packet encapsulated into new IP packet then enters into tunnel and travel through Internet until it reaches the tunneles exit point. Tunnel provides new routing functionalities using existing network infrastructure. A customer wants to use Detour forward its traffic to the nearest Detour router. The router then tunnel these packets to an exit point close to the destination within the Detour. Detour routers are edge devices in network. By taking alternate paths Detour try to improve latency and packet drop rate which automatically improves transport performance. Detour routers share information about measured latency, packet drop rate and available bandwidth along their each tunnel.

\subsection{G-ISP}

An additional ISP that provides transit services to its customers in an overlay network is called as Global ISP (GISP) [6]. G-ISP tries to solve or minimize inter domain routing problems such as slow convergence, lack of QoS and multicast support. A BGP connection is maintained between a G-ISP and its customer to learn the advertisement of routers. Then customer decides best paths for each network prefix using policy rules. There is no direct connectivity between G-ISP and its customer. A virtual link over multiple intermediate physical links and routers is maintained, whereas ISP and its customer have a direct link Because of no direct link between G-ISP and its customer

\section{Volume 5 Issue 1, January 2016}




\section{International Journal of Science and Research (IJSR) \\ ISSN (Online): 2319-7064 \\ Index Copernicus Value (2013): 6.14 | Impact Factor (2014): 5.611}

the IP tunneling that is IP in IP concept is used for data transfer. G-ISP paradigm provides shorter convergence time, end to end QoS support and inter AS multicast. G-ISP provides multicast by using unicast between G-ISP and its customers. Because of no direct link the G-ISP environment is scalable. The BGP connecting G-ISP and its customer can goes through ISP and possibly other intermediate ASs.

The customer use IP tunneling for routing through G-ISP this decides that packet travel through G-ISP route and not over ISP route. G-ISP provides two types of services to its customers that are unidirectional service and bidirectional service. In first case G-ISP process packets which are initiated by its customer. The content distributors use this service. So that response is decreased and some QoS is also provided. Second case G-ISP serve packets initiated and destined to its customer. The initiated packets are sent using IP tunneling and destined packets are serving by advertising multiple routes to destination.

\section{Conclusion}

Since Overlay enhance or add new functionality to existing network. There is a different overlay network; for each concentrating functionality which may depend on deployment of overlay or relay nodes and its interconnection. If we try to enhance existing Overlay we must rewrite it. But it increases cost. So there is a need to research on Overlay with multiple functionalities and changing its functionality is easy.

\section{References}

[1] Rami Cohen and Danny Raz," Cost Effective Resource Allocation Of Overlay Routing Relay Nodes", IEEE/ACM TRANSACTIONS ON NETWORKING, VOL. 22, NO. 2, APRIL 2014.

[2] Muthuselvi S., ParaylaShyry S., Ramachandran V., EmaldaRoslin S., NandhitaN.M.," A review on intelligent deployment of nodes for improving the performance of overlay network."

[3] H. Pucha and Y. C. Hu, "Overlay TCP: Multi-hop overlay transport for high throughput transfers in the Internet," Purdue University, West Lafayette, IN, USA, Tech. Rep., 2005.

[4] D. Andersen, H. Balakrishnan, F. Kaashoek, and R. Morris, "Resilient overlay networks," in Proc. 18th ACM SOSP, 2001, pp. 131-145.

[5] S. Savage, T. A. A. Aggarawl, T. Anderson, A. Aggarwal, D. Becker, N. Cardwell, A. Collins, E. Hoffman, J. Snell, A. Vahdat, G. Voelker, and J. Zahorjan, "Detour: A case for informed internet routing and transport," IEEE Micro, vol. 19, no. 1, pp. 50-59, Jan.-Feb. 1999.

[6] R. Cohen and A. Shochot, "The "global-ISP" paradigm," Comput.Netw., vol. 51, no. 8, pp. 1908-1921, 2007.

\section{Author Profile}

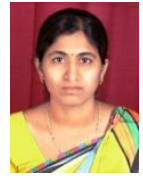

A. B. Jadhav received the B.E. degree in Computer Science and Engineering from M.S. Bidve Engineering College in 2010. Now, she is pursuing Master"s in Engineering (Computer Science and
Engineering) from M.S. Bidve Engineering college, Latur, SRTM University Nanded, Maharashtra.

N. G. Dharashive received the B.E. and M.E. degrees in Computer Science \& Engineering from M.B.E.Society"s College of Engineering, Ambajogai in 2001 and from Government College of Engineering, Aurangabad in 2011, respectively. He is pursuing Ph.D in Image Processing from S.R.T.M.University, Nanded (M.S.). He is now with M.S.Bidve Engineering College, Latur (M.S.) as Assistant Professor since 2002. 